LA-7568-N.

Informa Report

Beam Voltage and Current Parameter Study for the Autoresonant Acceleration Proof-of-Principle Experiment 
LA-7568-MS

Informal Report

UC-28

Issued: Novembe: 1978

\title{
Beam Voltage and Current Parameter Study for the Autoresonant Acceleration Proof-of-Principle Experiment
}

\author{
Brendan B. Godfrey
}

Rickey J. Fachl

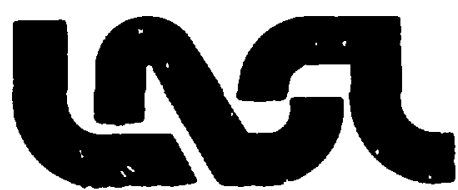


BEAM VOLTAGE AND CUKRENT PARAMETER STUDY FOR THE

AUTORESONANT ACCELERATION FROOF-OF-PRINCIPLE EXPERIMENT

by

Brendan B. Godfrey and Rickey J. Faehl

\begin{abstract}
ABS'RACT
Due to flashover problems, Aust in Research Associates, Inc. may be forced to perform its proof-of-principle autoresonant collective ion acceleration experiment at electron beam parameters of $2.25 \mathrm{MeV}$ and $15 \mathrm{kA}$ rather than the intended $3.0 \mathrm{MeV}$ and $30 \mathrm{kA}$. We show that the original experimental goals still can be achieved provided the beam radius is reduced by a factor of two and a thinner anode foil is employed. More generally, our parameter study suggests that operating with beams of smaller radii improves cyclotron wave behavior during beam adiabatic compression or expansion.
\end{abstract}

\title{
I. INTRODUCTION
}

The autoresonant collective ion acceleration proof-of-principle experiment, as proposed, ${ }^{1}$ was to accelerate protons to $30 \mathrm{MeV}$ using a 3-MeV, 30-kA electron beam. The acceleration process was to be controlled by varying the beam guide magnetic field from $25 \mathrm{~kg}$ to $2 \mathrm{~kg}$ over several meters. Radius of the beam at maximum magnetic field strength was to be $1 \mathrm{~cm}$. However, the electron beam generator has suffered per: istent electrical breakdown problems for several months. To ameliorate these difficulties, initial experiments probably will be performed in the $2.25-\mathrm{MeV}, 10$ - to $20-\mathrm{kA}$ range. 2

Previously we have made estimates of beam scatter by the ande foil, 3 cyclotron wave growth in a helix slow-wave amplifier, ${ }^{4,5}$ and wave behavior during ion acceleration. 6-8 The computations assumed a 3-MeV, 30-kA beam. Here, we repeat the analyses for several combinations of reduced beam energy, current, and radius. We find that increased scatter due to decreased beam 
vollage can be offset by use of thinner or less dense commercially available anode foils. Within reasonable limits, slow cyclotron wave growth in a helix amplifier is not strongly affected by electron energy and current. For the same beam radius and range of magnetic field strengths, the reduced current and voltage disastrously Increase the Ion acceleration length by a factor of 2.5 , on the other hand. Fortunately, it is necessary only to reduce the beam (and waveguide) radius by one-half in order to return the acceleration length to manageable proportions.

Section II treats foll scattering, Sec. III, wave length, and Sec. IV, ion acceleration. Few theoretical details are provided, since they are readily available in the aforementioned references. Some concluding observations art offered in Sec. $V$.

\section{I1. ANODE FOIL SCATTER}

Excessive angular scatter in the beam electron trajectories leads to rapid damping of cyclotron waves. This scatter varies adiabatically with guide magnetic field sirength. If the scatter is to be limited to $20^{\circ}$ at the front of the acceleration section, where $B_{z}^{0}=25 \mathrm{~kg}$, then the beam must leave the diode, where $B_{z}^{0}=2.5 \mathrm{~kg}$, with a scatter no greater than $6^{\circ}$. Anode foil induced scatter depends on the material and thickness of the anode foil and on the electron energy approximately as

$$
\theta=F^{1 / 2} / \beta^{2} \gamma
$$

where $F$ is obtained from Table $I$. The table of scattering coefficients was developed by $L$. E. Thode from Monte Carlo calculations. 3

Driginal experimental plans called for a 25.4- $\mu m(1-m i 1)$ titanium foll, which for $3 \mathrm{MeV}$ gives $5.4^{\circ}$. For $2.25 \mathrm{MeV}$, the value rises to 6.90. Evidently, a different foil is needed for the lower roltage. One possibility is $1 / 2$ mill. titanium, which gives $4.5^{\circ}$ at the lower energy. More generally, any foil with $F$ less than about 0.3 on Table $I$ is acceptable with respect to the $6^{\circ}$ scatter limit. Engineering considerations will bear heavily on the final choice.

\section{CYCLOTRON WAVE GROWTH}

Cyclotron waves required for autoresonant ion acceleration are to be first excited by an RF antenna and then anplified by a slow-wave structure. The helix 
amplifier has been thoroughly investigated for the original beam parameters and appears to be a good choice. The need for initially low phase velocities sets the helix pitch angle at $8^{\circ}$ for a $3.4-\mathrm{kg}$ magnetic guide field. The ratio of helix radius $\underline{R}$ to beam radius $\underline{a}$ is optimal at about 1.5 . With these helik parameters and the original beam parameters of $3.0 \mathrm{MeV}, 30 \mathrm{kA}$, and $2.65 \mathrm{~cm}$ radius, the wave e-folding distance is $34.4 \mathrm{~cm}$. Amplification by a factor of twenty can be achieved in a $1 \mathrm{~m}$ helix.

We have repeated these GRADR numerical calculations ${ }^{4-6}$ for a $2.25-\mathrm{MeV}$ beam of current 10,15 , or $20 \mathrm{kA}$ and a radius of 2.65 or $1.32 \mathrm{~cm}$. Table II gives wave frequency $\underline{w}$, wavenumber $\underline{k}$, and growth length $\underline{L}$ for various beam energies, currents, and radii. The e-folding length is seen to be relatively insensitive to beam parameters and in no case cited exceeds by $50 \%$ the $3-\mathrm{MeV}$ value. Wave growth appears to be no problem.

IV. ION ACCELERATION

The variation of wave properties and the corresponding increase in ion energy in the acceleration section is determined from conservation of wave energy flux and from the icn equations of motion. Figure 1 shows the change with position of the wave phase and group velocities, and accelerating 6 lectric field, the beam envelope motulation, the ion energy, the wave potential well depth, and the guide magnetic field. A he magnetic field decreases from 25.5 to $1.7 \mathrm{~kg}$. Potential depth and ion energy azs in $\mathrm{MV}$, the electric field in $\mathrm{MV} / \mathrm{cm}$, and the axial distance in $\mathrm{cm}$. The data is essentially that of Ref. 7 , but reformated. The amplitude of the cyclotion wave at the entrance to the acceleration region is set by $\delta a=a / 2$. Larger values of the beam envelope modulation are impractical. Ion energy reaches the desired $30 \mathrm{MeV}$ at $8.5 \mathrm{~m}$.

Figure 2 provides the same information for a 2.25-MeV, 15-kA electron beam, case 3 of Table II. Ions reach $30 \mathrm{MeV}$ at $21.5 \mathrm{~m}, 2.5$ times the Fig. 1 distance. A factor of two comes simply from cutting the total current in half. The remaining length increase is accounted for by the $20 \%$ increase in wave number at large $\mathrm{B}_{\mathrm{z}}^{0}$ for Fig. 2 relative to Fig. 1. Specifically, ka/2 is 1.56 as opposed to 1.31 at $\mathbf{B}_{z}^{0}=25.5 \mathrm{~kg}$. Other things being equal, ka $>2$ reduces the acceleration field sharply.

Results for the same $2.25-\mathrm{MeV}, 15-\mathrm{kA}$ beam but with beam and waveguide radius reduced by $1 / 2$, case 6 of Table II, are given in Fig. 3 . The distance needed to achieve $30-\mathrm{MeV}$ ions drops dramatically to $6.0 \mathrm{~m}$. The reduction 
TABLE I

FOIL SCATTERING FUNCTION $\theta^{2}=F / \beta^{4} \gamma^{2}$

$\underline{X(\mu \mathrm{m})} \quad \underline{127.0} \quad \underline{254.0} \quad \underline{508.0} \quad \underline{762.0} \quad \underline{1270.0} \quad \underline{2540.0}$

Deuterium

Tritium

0.00447

0.0114

0.0276

0.0455

0.0843

0.191

$50 / 50$

\begin{tabular}{|c|c|c|c|c|c|c|}
\hline$x(\mu \mathrm{m})$ & 12.7 & 25.4 & 50.8 & 76.2 & 127.0 & 254.0 \\
\hline Mylar & 0.0111 & 0.0292 & 0.0716 & 0.119 & 0.221 & 0.504 \\
\hline Kapton & 0.0115 & 0.0300 & 0.0735 & 0.122 & 0.227 & 0.517 \\
\hline Beryilium & 0.00944 & 0.0245 & 0.0597 & 0.0987 & 0.183 & 0.417 \\
\hline Graphite & 0.0211 & 0.0526 & 0.125 & 0.205 & 0.378 & 0.852 \\
\hline Aluminum & 0.0541 & 0.132 & 0.310 & 0.505 & 0.924 & 2.07 \\
\hline Titanium & 0.168 & 0.397 & 0.913 & 1.47 & 2.67 & 5.91 \\
\hline
\end{tabular}

TABLE II

SI,OW CYCLOTRON WAVE GROWTH IN AN $8^{\circ}$ HELIY, WITH $B_{2}=3.4 \mathrm{~kg}$ and $R / a=1.5$

\begin{tabular}{|c|c|c|c|c|c|}
\hline$v(\mathrm{MeV})$ & I (kA) & a $(\mathrm{cm})$ & $w_{0}\left(3.10^{10} \mathrm{~s}^{-1}\right)$ & $k\left(\mathrm{~cm}^{-1}\right)$ & $L(\mathrm{~cm})$ \\
\hline 3.0 & 30 & 2.65 & 0.060 & 0.40 & 34.4 \\
\hline 2.25 & 20 & 2.65 & 0.077 & 0.52 & 32.7 \\
\hline 2.25 & 15 & 2.65 & 0.075 & 0.50 & 38.9 \\
\hline 2.25 & 10 & 2.65 & 0.072 & 0.48 & 49.3 \\
\hline 2.25 & 20 & 1.32 & 0.079 & 0.48 & 30.8 \\
\hline 2.25 & 15 & 1.32 & 0.077 & 0.47 & 34.5 \\
\hline 2.25 & 10 & 1.32 & 0.076 & 0.47 & 42.8 \\
\hline
\end{tabular}


appears due solely to the smaller value of $\mathrm{ka} / 2$ at $z=0$, namely 0.76 . Also significant, the well depth at the start of acceleration is much greater, facilitating inicial ion trapping. We conclude, therefore, that decreasing the beam radius to about one-half the originally planned value is both necessary and sufficient for achieving the projected $30-\mathrm{MeV}$ ion energies at reduced beam energy and current.

As an interesting sidelight, we note that the ion energy corresponding to $\mathrm{B}_{z}^{0}=1.7 \mathrm{~kg}$ is $20 \%$ greater in Fig. 3 than in Fig. 2 . The difference is explained in terms of the approximate dispersion relation for slow cyclotron waves, 6

$$
w_{0}=k v-\left[w_{c}^{2} / \gamma^{2}-w_{p}^{2} / \gamma^{3}\right]^{1 / 2} .
$$

Roughly, the corresponding phase velocity squared is

$$
v_{\phi}^{2}=\left(w_{0} \gamma v / w_{c}\right)^{2} /\left(1-w_{p}^{2} / \gamma w_{c}^{2}\right)
$$

Decreasing the beam radius from Fig. 2 to Fig. 3 changes the denominator of (3) from about 0.95 to 0.80 . Thus, operating nearer the beam equilibrium limit enhances the variation of ion energy with magnetic field. It is, unfortunately, not clear that this observation has practical value.

\section{SUMMARY}

We have seen that electron beam reduced energy and current, due to generator problems, should have no significant impact on the proof-of-principle experiment provided beam radius is sufficiently reduced. Indeed, a $2.25-\mathrm{MeV}$, 15-kA beam injected into the acceleration region with $0.5-\mathrm{cm}$ radius gives a shorter ion acceleration length than does the original 3.0-MeV, 30-kA beam injected with a $1.0-\mathrm{cm}$ radius.

These specific numerical examples emphasize the general rule that ka/2 be less than unity to maximize acceleration rates. Even with the originally proposed experimental parameters, reduced beam radius would give better results. Admittedly, there are experimental problems associated with obtaining small diameter beams. Our numerical analyses nonetheless indicates that efforts in this direction should be made. 
This research was performed under the auspices of the US Department of Energy. Larry Havard of the US Army Ballistic Missile Defense Advanced Technology Center is the Project Manager.

\section{REFERENCES}

1. W. E. Drummond, G. I. Bourianoff, D. F. Brower, D. E. Hasti, W. W. Rienstra, M. L. Sloan, J. R. Thompson, J. R. Uglum, and H. V. Wong, "Proof of Principle Experiment for the Auto-Resonant Accelerator," I-ARA-76-U-100, Austin Research Associates, 1976.

2. M. L. Sloan, private communication, 1978 .

3. L. E. Thode, unpublished.

4. B. B. Godfrey, R. J. Faehl, B. S. Newberger, H. R. Shanahan, and L. E. Thode, "Relativistic Electron Beam Cyclotron Wave Growti in Helical Slow Wave Structures," in Proc. Second International Topica? Conference on High Power Electron and Ion Beam Research and Technology (Cornell Univ., Ithaca, 1977), Vol. II, P. 541.

5. R. J. Faeh1, B. S. Newberger, and B. B. Godfrey, "Simulation of Cyclotron Wave Growth in a Helical Slow Wave Structure," Phys. Fluids, to be published.

6. B. B. Godfrey, "Linear Theory of Radially Inhomogeneous Unneutralized Relativistic Electron Beams," submitted to IEEF Trans. on Plas. Sci.

7. B. B. Godfrey and B. S. Newberger, "Wave Amplitude Variation and Energy Flow in Autoresonant Collective Ion Acceleration," J. Appl. Phys., to be published.

8. R. J. Faehl, W. R. Shanahan, and B. B. Godfrey, "Nonlinear Characteristics of Cyclotron Waves in an ARA Configuration," in Proc. Third International Conference on Collective Methods of Acceleration (Univ. California, Irvine, 1978), to be published. 

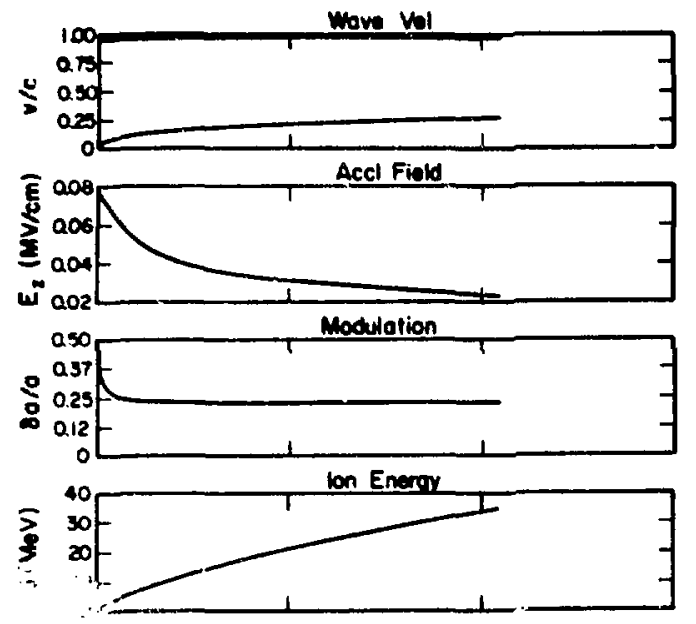

Polentiol
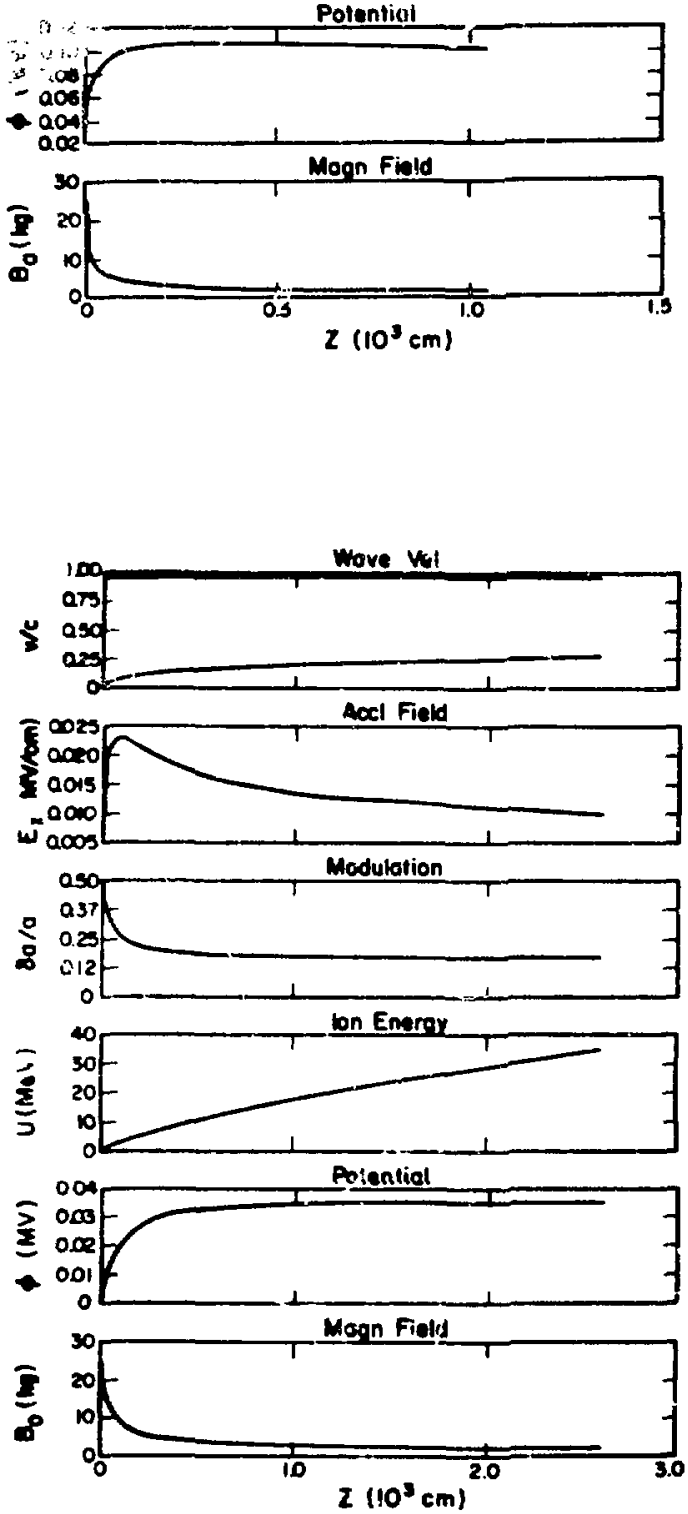

Fig. 1. Variation of wave parameters and beam energy in the acceleration section of the proof-of-principle auroresonant acceleration experiment for a $3-\mathrm{MeV}, 30-\mathrm{kA}$ electron beam injected at the left with a $1.0 \mathrm{~cm}$ radius. Wave frequency is $w_{0}=1.8010^{9} \mathrm{~s}^{-1}$.
Fig. 2, Variation of wave parameters and beam energy in the acceleration section of the proof-of-principle autoresonant acreleration experiment for a $2.25-\mathrm{MeV}, 15-\mathrm{kA}$ electron beam injected at the left with a $1.0 \mathrm{~cm}$ radius. Wave frequency is $2.2510^{9} \mathrm{~s}^{-1}$. 

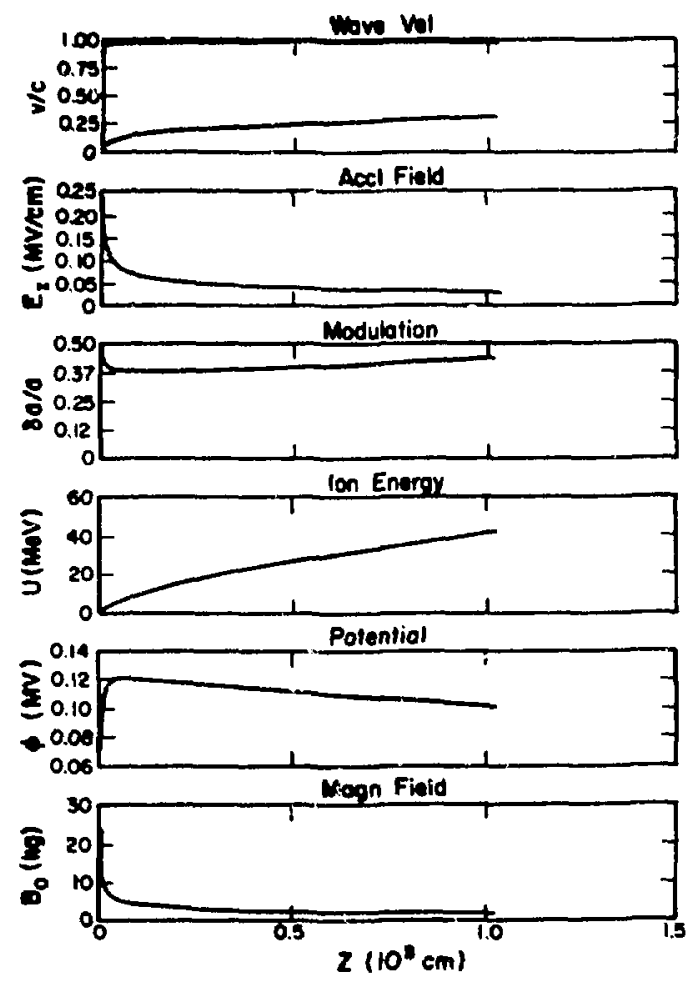

Fig. 3. Variation of wave parameters and beam energy in the acceleration section of the proof-of-principle autoresonant acceleration experiment for a $2.25-\mathrm{MeV}, 15-\mathrm{kA}$ electron beam injected at the left with a $0.5 \mathrm{~cm}$ radius. Wave frequency is $2.3110^{9} \mathrm{~s}^{-1}$. 